DOI: 10.17951/en.2019.4.179-194

\begin{tabular}{lcr}
\hline & ANNALES \\
& UNIVERSITATIS MARIAE CURIE-SKŁODOWSKA & \\
LUBLIN - POLONIA & \\
VOL. IV & SECTIO N & 2019 \\
\hline
\end{tabular}

Ilona Feld-Knapp

Lorand Universität Budapest, Ungarn

ORCID: https://orcid.org/0000-0002-2612-5683

knappilona@t-online.hu

Alessa Weimann

Freie Universität Berlin, Deutschland

ORCID: https://orcid.org/0000-0001-7463-7969

alessa.weimann@gmx.de

\title{
Instrumente für die Reflexion. Zur Funktion und Erstellung von Reflexionsinstrumenten im universitären Kontext
}

\author{
Instrumenty refleksji. Tworzenie instrumentów \\ służących refleksji w kontekście kształcenia \\ uniwersyteckiego oraz ich funkcje
}

Resümee: In dem Beitrag werden die Erfahrung und die Zusammenarbeit der Autorinnen zusammengefasst dargestellt. Im Fokus stehen dabei die erstellten Instrumente zur Reflexion und wie diese im universitären Kontext gewinnbringend eingesetzt werden können. Es handelt sich dabei um einen Feedbackbogen, der Kriterien für eine erfolgreiche Präsentation beinhaltet und um einen Beobachtungsbogen für den Unterricht, der Lehramtsstudierende auf ihrem Weg zur professionellen Unterrichtsbeobachtung unterstützen soll. Es wird außerdem auf die Seminare, bei denen die Idee zu den Projekten entstanden ist und auf die Funktionen von Reflexion im Allgemeinen eingegangen.

Schlüsselwörter: Feedback; Unterrichtsbeobachtung; Projektseminar; Hospitation; Bewertungskriterien 


\section{EINLEITUNG}

Die Idee zu dem vorliegenden Artikel entstand aus der Zusammenarbeit der beiden Autorinnen im Rahmen eines von Alessa Weimann absolvierten Praktikums, welches im Rahmen des Masterstudiengangs „Deutsch als Fremdsprache: Kulturvermittlung " an der Freien Universität Berlin am Lehrstuhl für Sprachpraxis und Fachdidaktik am Germanistischen Institut der ELTE Universität Budapest stattfand. Betreut wurde das Praktikum von Professor Dr. habil. Ilona Feld-Knapp, der langjährigen Leiterin dieses Lehrstuhls. Die Zusammenarbeit der beiden Universitäten blickt auf eine längere Tradition zurück, regelmäßig haben Studierende der FU Berlin die Möglichkeit, ihre Fähigkeiten in der Hauptstadt Ungarns zu vertiefen.

Die Ziele des Praktikums sind unter anderem die Vertiefung der fachwissenschaftlichen Kenntnisse, insbesondere in der Fachdidaktik, sowie eine Erhöhung der interkulturellen Kompetenz durch kulturelle Fremderfahrungen. Dies soll durch das Kennenlernen unterschiedlicher Lehr- und Lernsituationen geschehen. Es werden innerhalb des Praktikums vielfältige Angebote und Erfahrungen möglich gemacht. In diesem Zusammenhang konnte ein kommunikationsfördernder Kurs zum Thema „Landeskunde“ am Eötvös József Collegium übernommen werden. In dem Kontext des Praktikums wurden außerdem fachdidaktische Seminare und ein Projektseminar besucht, in denen neben der Hospitation auch konkrete Aufgabenbereiche übernommen wurden, die die eigene Lehrkompetenz förderten.

Bei diesen Aufgabenbereichen handelte es sich um die Entwicklung eigener Materialien. Hier entstanden nach enger Zusammenarbeit und Absprache zwei Instrumente mit unterschiedlichen Perspektiven. Zum einen wurde ein Feedbackbogen für die Evaluation von Präsentationen konstruiert, mit dem Präsentationen nach bestimmten Kriterien reflektiert werden können und welcher während des Seminars bereits erprobt werden konnte. Zum anderen ging aus dem Austausch die Erstellung eines auf die Teilnehmenden bzw. das Thema eines Seminars zugeschnittenen Beobachtungsbogens für den Unterricht hervor. Innerhalb dieses Seminars wurden verschiedene Schulen besucht und Schulstunden beobachtet. Damit in den Hospitationen der Blick auf bestimmte Abläufe des Unterrichts gelenkt und die Reflexion erleichtert wird, entstand nach ausgiebiger Recherche das genannte Instrument.

Im Folgenden sollen nach der ausführlichen Vorstellung der Unterrichtssituationen und der Studierenden sowie dem Bezug auf die Fachliteratur die beiden entstandenen Instrumente detailliert vorgestellt werden. Dabei wird außerdem auf den Ausbau des Wortschatzes eingegangen, da die Studierenden in der Regel Deutsch nicht als Muttersprache erlernt haben. 
VORAUSSETZUNGEN UND HINTERGRÜNDE

In diesem Abschnitt werden zunächst die Seminare und Studierenden genauer beschrieben und anschließend werden die Hospitationen an den Musterschulen kurz umrissen, um die Hintergründe der Entstehung der vorzustellenden Instrumente zu verdeutlichen.

Zunächst wird das Projektseminar, in dem der Feedbackbogen erprobt wurde, dargestellt. Dieses Seminar fand im Herbstsemester 2018/2019 statt. Bei den 18 ungarischen Studierenden (13 Studentinnen, 5 Studenten) handelte es sich um Germanistikstudierende in ihrem 3. Studienjahr, die sich sprachlich auf dem Niveau B2/C1 befinden sollten. In der Praxis waren allerdings kleinere Mängel im Sprachgebrauch zu erkennen. Das Seminar schloss das Modul „Textkompetenz und Sprachenlernen“ ab. Das Modul besteht aus einer einführenden Vorlesung, darauf bauen zwei Folgeseminare auf. Das eine Seminar stellt die Präsentations- und Kommunikationskompetenz in den Fokus und das andere ist das bereits oben genannte Projektseminar. Ziel des Projektseminars war die intensive Auseinandersetzung mit Literaturpreisen und Festspielen sowohl im deutschsprachigen als auch ungarischen Raum. Dadurch sollte die Interkulturalität gefördert werden und die Kenntnis der jeweiligen Landeskunde vertieft werden. Hierzu wurde zunächst ein theoretischer Text zur Textkompetenz (Portmann-Tselikas und Schmölzer-Eibinger, 2008) aufbereitet, damit ein besseres Verständnis für die spätere Arbeit mit den verschiedenen Texten gesichert werden konnte. Außerdem wurden Literaturpreise und Festspiele exemplarisch für Berlin und Ungarn von den Kursleiterinnen vorgestellt, um die Studierenden mit der Textarbeit vertraut zu machen und den Austausch über die Texte zu fördern. Währenddessen wurde immer wieder der Fokus auf den Ausbau des Wortschatzes der Teilnehmenden gelegt. Dies wurde mithilfe erstellter Arbeitsbögen durchgeführt, die die Wortschatzvarianz in Bezug auf thematisch passendes Vokabular förderten. Im Anschluss sollten die Studierenden nach mehrstündiger Recherchearbeit ihre eigenen Ergebnisse in drei vorher festgelegten Gruppen vorstellen. Eine Gruppe war für jeweils ein deutschsprachiges Land zuständig, so gab es eine Deutschland-Gruppe, eine Österreich-Gruppe und eine Schweiz-Gruppe. Jede Gruppe hatte eine Stunde Zeit, um ihre Informationen vorzustellen. Währenddessen wurde der Vortrag mithilfe des erstellten Feedbackbogens beobachtet, mit besonderem Fokus auf dem Sprachgebrauch und der inhaltlichen Struktur. Im Anschluss wurde ein mündliches Feedback gegeben. Hervorzuheben ist die Akzeptanz der Kritik von den Studierenden, Verbesserungsvorschläge wurden ernst genommen und sowohl inhaltlich als auch sprachlich in den nächsten Präsentationen umgesetzt. 
Abgeschlossen wurde das Projektseminar mit ,Messeständen', bei denen die gesammelten Informationen der Präsentationen noch weiter ausgebaut werden sollten, beispielsweise mit ergänzenden Artikeln, Programmheften etc.

Der andere, ebenfalls im Herbstsemester 2018/2019 stattfindende Kurs, bei dem die Idee zur Gestaltung eines eigenen Beobachtungsbogens für den Unterricht entstand, behandelte aktuelle Fragen der Fachdidaktik. Die 16 Teilnehmenden (10 Studentinnen, 6 Studenten) waren ungarische Lehramtsstudierende in ihrem 4. Studienjahr. Innerhalb des Kurses herrschte eine produktive, motivierte Stimmung, der Austausch über die Fachliteratur und die eigenen Präsentationen bzw. Ausarbeitungen wurde gefördert. Es wurde sich intensiv mit dem Thema der Textkompetenz auseinandergesetzt, sie stand im Fokus des Seminars und auf ihr bauten sowohl die Hospitationen als auch die eigens erstellten Materialien zu einer textbasierten Einheit, die am Ende des Seminars in Gruppen vorgestellt wurden. Auch zu diesen Präsentationen wurde mündliches Feedback gegeben, wobei der Fokus hier weniger auf der Sprache als auf dem Inhalt und der Vortragsweise lag. Die beiden Hospitationen fanden mit einem zweiwöchigen Abstand an den Übungsschulen der Universität statt. Jeweils eine Unterrichtseinheit wurde an dem Eötvös József Gymnasium und dem ELTE Trefort Ágoston Gymnasium absolviert. Hier sollte der Unterricht mit Blick auf die Förderung der Textkompetenz beobachtet werden. Mit diesen Hospitationen im Hinterkopf ist der noch vorzustellende Beobachtungsbogen entstanden.

\section{FUNKTIONEN VON FEEDBACK}

Im Folgenden wird zunächst die Funktion von Feedback erläutert, im Anschluss wird auf Beobachtungen in der Schule eingegangen.

Die Notwendigkeit von Feedback ist unumstritten, bereits in der Schule bekommt man Rückmeldungen über seine Leistungen, im universitären Kontext ebenso. Doch auch im Arbeitsumfeld, und dabei besonders in der Funktion eines Lehrenden, ist man ständig mit Feedback konfrontiert. Feedback wird dabei definiert als eine Mitteilung bzw. Rückmeldung „an eine Person, die darüber informiert wird, wie ihre Verhaltensweise von jemand anderem wahrgenommen, verstanden und erlebt wird“ (Weber, 2012, S. 3). Diese „bewusst herbeigeführte Gesprächssituation“ (Noltenius, 2012, S. 12) kann dabei von einer gleichgestellten Person (Mitschüler/in, Kommilitone/in, Arbeitskollege/ in) oder einer höhergestellten Person (Lehrkraft, Vorgesetzter etc.) oder anders herum vorgenommen werden. „Dabei wird der Feedback-Nehmer mit den [unter Umständen subjektiven] Beobachtungen der Feedback-Geber zum eigenen 
Verhalten konfrontiert" (Noltenius, 2012, S. 12), d.h. Feedback bekommen bietet eine Chance, das eigene Verhalten durch konstruktive Kritik zu optimieren.

Dieses Auseinandersetzen mit anderen Meinungen führt zur Professionalisierung. Dementsprechend wird Feedback geben besonders im universitären Kontext nach Präsentationen praktiziert. Präsentieren und Feedback geben/annehmen verstehen sich dabei als Schlüsselkompetenzen, also „überfachliche Kompetenzen“ (Brunner, 2010, S. 750). Bei einem professionellen Feedback ist darauf zu achten, dass es hilfreich für den Empfänger und dabei konkret, gezielt, verständlich und begründet ist (vgl. Weber, 2012, S. 3-4). Reflektiert der Feedback-Nehmer das Gehörte, kann die Selbstwahrnehmung und die eigene (Präsentations-)Kompetenz verbessert werden. Damit das erteilte Feedback nicht wahllos, unstrukturiert und dadurch besonders in mündlicher Mitteilungsform nicht eingängig oder im schlimmsten Fall unverständlich ist, kann ein Feedbackbogen helfen bzw. unterstützend wirken. Online lassen sich zahlreiche Variationen solcher Bögen finden, in Kapitel 4.1 wird eine eigens erstellte und erprobte Möglichkeit vorgestellt.

Hospitationen und die intensive Beobachtung dieser sind ein fester Bestandteil in der Lehrerausbildung. Sie fördern die Entwicklung der eigenen Lehrkompetenz. Die Unterrichtsbeobachtung ist Teil verschiedener Forschungsdisziplinen, unter anderem "gehört [sie] auch zu den zentralen Ausbildungs- und Forschungsverfahren für Deutsch als Fremd- und Zweitsprache" (Krumm, 2010, S. 1363). Dabei wird das Verhalten der Lehrenden und Lernenden in einem Dreischritt beobachtet, analysiert und meistens auch in einer Form beurteilt bzw. reflektiert (Krumm, 2010, S. 1363). Besonders in der Lehrerausbildung wird dieses Verfahren genutzt, um zu lernen, den Unterricht bewusst wahrzunehmen, zu reflektieren und auf späteres eigenes Handeln im Unterricht zu übertragen. „In der Lehrerausbildung wird die Beobachtung meist mit fachdidaktischen Seminaren gekoppelt und stellt die Vorstufe zu eigenen Unterrichtsversuchen angehender Lehrer dar" (Krumm, 2010, S. 1365), das heißt, der Fokus der Beobachtung richtet sich nach dem Schwerpunkt des Seminars. Dementsprechend gibt es keine allgemeingültigen Beobachtungskriterien, die Kategorien bzw. Kriterien für die Beobachtung ändern sich nach Forschungsund Erkenntnisinteresse (vgl. Krumm, 2010, S. 1365). Die Frage, welche dieser Kategorien sich eignen, um den Unterricht zielgerichtet beobachten zu können, kann, wenn zeitlich umsetzbar, was in dem beschriebenen Seminar nicht möglich war, Teil des Seminars sein und von den Studierenden eigenständig ausgearbeitet werden.

Laut De Boer und Reh ist „die Schulung eines beobachtenden Blicks notwendig, um eine Sensibilisierung in der Wahrnehmung, ein Bewusstsein für die 
sprachliche Darstellung pädagogischer Situationen und schulischer Kontexte sowie eine Steigerung von Selbstbeobachtung und -reflexion zu erreichen" (De Boer und Reh, 2012, S. V). Diese Schulung muss bereits in der Ausbildung gefördert werden, wobei deutlich gemacht werden muss, dass Beobachtungen immer subjektiv, deutend und wertend sind (De Boer und Reh, 2012, S. V). Es geht bei der Beobachtung nicht um die Beurteilung der Persönlichkeit der Lehrenden und Lernenden, sondern um die Reflexion der Fähigkeiten und das Erkennen übergreifender Zusammenhänge und Lernziele. Perspektivenwechsel soll gefördert werden. Die Verfahren der Beobachtung und Reflexion sollten nicht nur in der Lehrerausbildung gefördert, sondern auch in der beruflichen Zukunft immer wieder praktiziert werden (De Boer und Reh, 2012, S. VI).

Für die Erstellung eines Beobachtungsbogens ist auf verschiedene Dinge zu achten. Eine Beobachtung kann keinen Anspruch auf Vollständigkeit haben, nicht alles kann zeitgleich wahrgenommen werden, da „schulische Situationen [...] hoch komplex [sind und] zahlreiche Interaktionen unterschiedlicher Personen [...] zeitgleich [ablaufen]" (De Boer und Reh, 2012, S. 70). Dementsprechend sind, wie bereits weiter oben erwähnt, passende Kriterien nötig, um den Blick gezielt auf ein Phänomen zu lenken und das Verstehen zu fördern. Das theoretische Vorwissen, welches die Studierenden in ihrer Lehrerausbildung erwerben, kann den Untersuchungsgegenstand und die darauffolgende Interpretation maßgeblich beeinflussen (vgl. Bennewitz, 2012, S. 206). Dabei muss darauf geachtet werden, dass der „Fokus der Beobachtung nicht zu stark einzuengen“ (Bennewitz, 2012, S. 206) ist. Durch verschiedene Kriterien können in der späteren Analyse (situationsbedingte) Zusammenhänge, Abhängigkeiten im Kontext verstanden und reflektiert werden. In Kapitel 4.2 wird ein auf das Seminar passender Beobachtungsbogen und die gewählten Kriterien ausführlich dargestellt. Dabei wird allerdings auf eine tatsächliche Analyse der hospitierten Unterrichtsstunde verzichtet.

\section{VORSTELLUNG DER ENTWICKELTEN MATERIALIEN}

Die im Folgenden darzustellenden Materialien sind das Ergebnis der Zusammenarbeit der beiden Autorinnen. Als Praktikantin bekommt man vielfältige Angebote zur Vertiefung der eigenen Kompetenzen, wobei die Erstellung der Reflexionsinstrumente ein solches Angebot darstellt. Dabei wurden schon vorhandene Beobachtungsbögen sowie Fachliteratur verwendet und diese weiter ausgebaut und vertieft. Die Vorschläge der Praktikantin wurden gemeinsam reflektiert und die Ergebnisse werden nun dargestellt. 


\section{Feedbackbogen für Präsentationen}

Innerhalb dieses Teilkapitels werden die ausgewählten Kategorien des Feedbackbogens (siehe Anhang 1) dargestellt und welche Funktionen diese haben. Außerdem soll gezeigt werden, wie der Bogen einen sprachlichen Beitrag leisten kann und wie er erweitert werden könnte. Des Weiteren soll auf die Annahme bzw. Akzeptanz des Feedbacks der Studierenden eingegangen werden.

Der „Feedbackbogen - Kriterienkatalog für eine erfolgreiche Präsentation“ ist unterteilt in drei grobe Themen: den Inhalt, die Sprache und das Auftreten und die Vermittlung. Jedes dieser Themen hat 5-6 Kategorien. Zu jeder Kategorie gibt es eine kurze Beschreibung, die verdeutlicht, was mit dem Begriff gemeint ist und was für eine sehr gute Leistung zu erwarten ist. In den letzten fünf Spalten kann die eigene Bewertung eingetragen werden $(++,+, /,-,--)$, welche mit den ungarischen Noten 5-1 übereinstimmt. Anzumerken ist hier, dass der Kriterienkatalog nicht für eine benotete Bewertung ist, sondern lediglich als Hinweis, als Feedback für die Präsentierenden gilt, dementsprechend werden Zeichen zur Bewertung genutzt. Der untere Teil des Bogens kann für eigene Notizen genutzt werden. Dieser Feedbackbogen orientiert sich an anderen Bögen dieser Art, dessen Links im Anhang zu finden sind.

Der erste Themenkomplex „Inhalt“ ist in Thema, Einleitung, Struktur/ Aufbau, Qualität, Quantität und Fragen gegliedert. Da das Thema bereits festgelegt wurde, kann dieses Kriterium abgewandelt werden in „Wie wurde das gegebene Thema umgesetzt?". Das Ergebnis aus den sechs Kategorien schließt den Komplex ab. Da es sich um drei einstündige Präsentationen mit je fünf Personen handelte, die ihre Arbeit zu Literaturpreisen und Festspielen präsentierten, wurden viele Informationen vorgetragen. Dementsprechend war die Struktur ein für das Feedback besonders wichtiger Punkt.

Der zweite Komplex „Sprache und Auftreten“ legt den Fokus auf den bzw. die Präsentierenden und wie dessen bzw. ihr Verhalten und sprachliches Vermögen sich auf den Vortrag auswirken. Dabei handelt es sich um das Ausdrucksvermögen, das Sprechtempo, den Redefluss, die Gestik/Haltung und den Blickkontakt. Drei der fünf Kriterien basieren auf der Sprache bzw. dem Sprachgebrauch, da dies besonders bei Nichtmuttersprachlern überprüft werden muss, um auf mögliche Schwachstellen aufmerksam zu machen. Bei den Studierenden, bei denen dieser Bogen erprobt wurde, handelte es sich um Lehrer in der Ausbildung und um Germanistikstudierende, d.h. bei beiden Studienfächern ist ausgezeichneter Sprachgebrauch von Nöten und muss immer wieder, auch auf einem bereits sehr hohen Niveau, gefördert werden. Dabei geht es hauptsächlich um Wortschatzerweiterung, aber auch einige grammatikalische Fehler, wie falscher 
Artikelgebrauch und dadurch falsche Kasuszuweisung sowie falscher Satzbau, kommen vor. Hierbei ist zu berücksichtigen, dass eine Präsentation mündlich erfolgt und sich dadurch Flüchtigkeitsfehler einschleichen können, nichtsdestotrotz müssen diese den Studierenden bewusst gemacht werden.

Der letzte Komplex „Vermittlung“ bezieht sich auf die Art und Weise der Präsentation. Hierbei werden der Medieneinsatz, die Kreativität, die Visualisierung, das Handout und gegebenenfalls die Teamarbeit betrachtet. Bei den zu bewertenden Gruppen war der Medieneinsatz frei wählbar, es entschieden sich trotzdem alle Teilnehmenden für eine Präsentation mit PowerPoint sowie ein unterstützendes Plakat. Anhand des Plakats wurde die Kreativität deutlich. Die Visualisierung wurde durch Videos und Bilder unterstützt. Ein Handout wurde bei den ersten Präsentationen nicht mitgereicht, was allerdings bei einem Umfang von knapp 60 Minuten nötig gewesen wäre. Nachdem dies kritisiert wurde, wurde ein Handout für die letzte Präsentation erstellt und die anderen Gruppen reichten ihre Ausarbeitung in der letzten Einheit nach. Da es sich bei diesem Seminar um eine Projektarbeit handelte, stand die Teamarbeit im Fokus und wurde von Anfang des Semesters an gefördert. Dementsprechend floss die Zusammenarbeit in das Feedback mit ein.

Was die sprachliche Gestaltung des Feedbackbogens betrifft, so ist anzumerken, dass nur eine positive Beschreibung der einzelnen Kriterien erfolgt. Dies ist bewusst so geschehen, erstens, da es sinnvoll ist, mit einem positiven Feedback zu beginnen und dieses hervorzuheben. Zweitens stellen diese Kriterien den Idealfall dar, sind sie erfüllt, kann die Leistung mit einem „++“ bewertet werden. Alles, was dem nicht mehr entspricht, muss in Abstufungen niedriger bewertet werden. Durch den fehlenden negativen Teil der Kriterien könnte sich eine Aufgabe für die Teilnehmenden ergeben, die den Wortschatz fördert, in dem sie die Gegenteile finden. Außerdem könnte eine weitere Aufgabe sein, die Studierenden ein schriftliches Feedback nach dem Vorbild des Bogens schreiben lassen.

Insgesamt erwies sich die Erstellung des Feedbackbogens als sinnvoll und lehrreich, sowohl für die Praktikantin als auch die Studierenden. Das Feedback erfolgte nach gleichen Mustern, der gleichen Struktur und war so für alle nachvollziehbar und gleichberechtigt. Durch die Fremdperspektive der Praktikantin als Muttersprachlerin konnte ein authentisches Feedback in Bezug auf die Sprache erfolgen, welches auch angenommen wurde. Nach jedem Vortrag wurde den Studierenden ein mündliches Feedback auf Basis des Feedbackbogens gegeben, dementsprechend bekam jeder eine persönliche Rückmeldung über seine "Sprache und Auftreten“ und ein gebündeltes Feedback zu „Inhalt" und „Vermittlung“. Dass dieses Feedback akzeptiert wurde, war daran erkenntlich, 
dass in den weiteren Vorträgen bzw. den abschließenden Messeständen auf die Kritik reagiert wurde und Fehler sowie Ratschläge umgesetzt wurden. So wurde klarer und langsamer gesprochen, die Folien der Präsentation übersichtlicher gestaltet und besonders auf die sprachliche Richtigkeit wurde Wert gelegt. Wie in Kapitel 2 erwähnt, wurden einige Wortschatzübungen in dem Kurs durchgeführt, dessen Varianten dann in den Vorträgen genutzt wurden.

Die Erstellung des Feedbackbogens war, trotz zahlreicher Vorlagen, die man einfach hätte übernehmen können, eine die eigene Kompetenz fördernde Aufgabe. Entstanden ist so ein Produkt, das auch für den weiteren beruflichen Gebrauch von Nutzen sein kann. Der Kriterienkatalog kann durch seine Allgemeingültigkeit sowohl als Handreichung für Lehrkräfte als auch Studierende bzw. Lernende dienen. Für Studierende kann dieser Bogen ebenfalls als Leitfaden bzw. Überprüfung der eigenen Leistung vor dem eigentlichen Vortrag dienen.

\section{Beobachtungsbogen für den Schulbesuch}

In diesem Teilkapitel wird der Beobachtungsbogen (siehe Anhang 2) ausführlich dargestellt. Es wird auf die Wahl und Nützlichkeit der Kriterien eingegangen. Außerdem soll gezeigt werden, wie der Bogen einen Beitrag zur Entwicklung der Lehrkompetenzen haben kann.

Der „Beobachtungsbogen für den Unterricht“ ist in sieben Spalten aufgeteilt. Die erste Spalte beschreibt das zu beobachtende Kriterium, die zweite Spalte ist zum Festhalten der eigenen Beobachtungen und Ergänzungen vorgesehen und die letzten vier Spalten wurden für die Bewertung erstellt. Bewertung meint hier eher eine Übersicht schaffen, als das Beobachtete zu beurteilen. Die Zeichen ,,+++- und $\varnothing$ bedeuten hierbei: häufig beobachtet, beobachtet, fehlte und nicht Teil der Beobachtung. Der Beobachtungsbogen ist in sieben Zeilen geteilt, die bestimmte Kriterien in den Fokus nehmen: das Unterrichtsklima und die Unterrichtsführung, die Gesprächsformen und -gewichtung, die Zielorientierung und Struktur, die Aktivierung und Motivierung, die Textarbeit, die Variabilität der Unterrichtsformen und die Lernerfolgssicherung. Jedes dieser Kriterien hat zwei bis fünf Unterpunkte, die bestimmte Aspekte, die im Unterricht als wichtig zu erachten sind, hervorheben.

Der erste Kriterien-Komplex „Unterrichtsklima und -führung“ beschreibt die Rahmenbedingungen des Unterrichts. Es wird nach einer störungsfreien Arbeitsatmosphäre, die im Umkehrschluss ein hohes Maß an tatsächlicher Lernzeit bedeutet, gefragt. Außerdem soll das Unterrichtstempo und der Umgang mit Fehlern beobachtet werden. Ein weiterer Fokus liegt auf dem Umgang miteinander, sowohl das Verhältnis zwischen Lehrkraft und SchülerInnen als auch 
das Miteinander der Lernenden soll untersucht werden. Dieser erste Komplex bedingt den Verlauf des Unterrichts erheblich, weshalb eine ausführliche Beobachtung lohnenswert ist.

Das zweite Kriterium ist „Gesprächsformen und -gewichtung“, hierbei sollen die genutzten Gesprächsformen beobachtet werden. Abwechslungsreiche Redeanlässe und Sozialformen gestalten den Unterricht interessanter und anspruchsvoller. Außerdem soll verfolgt werden, ob jeder der Lernenden zu Wort kommt und ob der Austausch gefördert wird. Dadurch kann die Flexibilität der Lehrkraft beurteilt werden: ist ein Eingehen auf Fragen möglich und wird dazu ermutigt oder wird zu sehr am erstellten Plan festgehalten? Ein weiterer Beobachtungspunkt ist die Lehrkraft-SchülerInnen-Gewichtung, hierbei ist zu verfolgen, ob sich die Lehrkraft zurücknimmt und ob versucht wird, hauptsächlich den Lernenden das Wort zu übergeben. Es ist ein allgemeines Problem, dass im (Fremdsprachen-)Unterricht der Sprechanteil der Lehrenden zu hoch ist (vgl. Brinitzer, Hantschel, Kroemer, Möller-Frorath und Ros, 2016, S. 12). Es sollte stattdessen authentisches Sprechen bei den Lernenden unterstützt werden. Die ausführliche Beobachtung kann Aufschluss über mögliche Probleme geben.

Die nächste Zeile nimmt die "Zielorientierung/Struktur" in den Fokus. Hierbei soll auf die Klarheit der Struktur und Aufgabenstellungen geachtet werden. Gibt es hier Mängel, kann es zu Verwirrung und damit zu Verzögerungen im Ablauf kommen. Auch die Verständlichkeit und Transparenz in Bezug auf die Lernziele der Lehrkraft ist ein wichtiges Kriterium für eine reibungslose Unterrichtsstunde, sinnvolle Übergänge schaffen Kohärenz.

„Aktivierung und Motivierung“ werden als nächstes in den Mittelpunkt gestellt. Die SchülerInnen sollen aktiv und selbstständig am Unterrichtsgeschehen teilnehmen, dabei sollen die Leistungserwartungen weder zu über-noch zu unterfordernd sein. Eine weitere Beobachtungsfrage geht dem Abwechslungsreichtum nach: kann das Interesse und die Kompetenz der Lernenden gefördert werden? Hierbei können auch die Kreativität und das Eingehen auf Interessen der SchülerInnen beurteilt werden. Die Motivation und aktive Teilnahme der Lernenden sind wichtige Faktoren für einen erfolgreichen Unterricht.

Als nächstes wendet sich der Beobachtungsbogen der „Textarbeit“ zu. Dieser Teil wurde eingefügt, da er Thema des Seminars war und die Studierenden das erlernte Wissen auch in den Hospitationen wiederfinden sollten. Hierbei soll auf den Einbezug von verschiedenen Texten und Textsorten geachtet werden. Unterschiedliche Texte fordern unterschiedliche Strategien, die im Unterricht geübt werden können. So werden „durch Sachtexte [...] vor allem informierende, alltagsbezogene und landeskundliche Themen angeboten. Literarische Texte [...] sprechen die Lernenden ästhetisch und affektiv an und ermöglichen eher 
erzieherische Lehr- und Lernziele, die über den reinen Spracherwerb hinausweisen und zur Persönlichkeitsentwicklung der Lernenden beitragen" (Feld-Knapp, 2016, p. 298). Hierbei muss auf die passende Auswahl geachtet werden: ist der Text inhaltlich und sprachlich angemessen, können die Lernenden einen Mehrwert aus dem Text ziehen? Bei dem Aufbau der Stunde sollte außerdem darauf geachtet werden, ob und inwiefern das 3-Phasen-Modell im Ablauf zu erkennen ist. Das kann als Input für die spätere eigene Arbeit wichtig sein. Außerdem wird in diesem Abschnitt nach ,vom Text zum Text' gefragt. Gemeint ist damit, ob eine eigene Textproduktion von den SchülerInnen gefordert wird und wenn ja, wie diese umgesetzt wird.

Anschließend rückt die „Variabilität der Unterrichtsformen“ in den Fokus der Beobachtung. Hierbei soll auf unterschiedliche methodische Vorgehensweisen bzw. Sozialformen geachtet werden. Außerdem ist zu beobachten, wie abwechslungsreich und damit spannend der Unterricht in Bezug auf die Medienvarianz gestaltet ist.

Abgeschlossen wird der Beobachtungsbogen mit der „Lernerfolgssicherung“ - im Fokus steht hier die Ergebnissicherung. Zu beobachten ist, wie die Ergebnisse der Stunde besprochen und festgehalten werden. Des Weiteren sollen die Hausaufgaben auf ihre sinnvolle und ergänzende Funktion überprüft werden.

Anzumerken ist an dieser Stelle, dass in der Darstellung des Beobachtungsbogens chronologisch vorgegangen wurde und jedes Kriterium nacheinander in den Fokus der Erläuterung gestellt wurde. Während einer Hospitation muss allerdings auf viele der Punkte gleichzeitig geachtet werden, welches eine Herausforderung für den Beobachter darstellt, weshalb das professionelle Beobachten immer wieder geübt werden sollte. Dementsprechend muss sich vorher überlegt werden, welche der Kriterien besonders verfolgt werden soll. In dem Fall des bereits besprochenen Seminars wäre es die Textarbeit gewesen.

Mit dem Beobachtungsbogen soll der Blick der Studierenden auf einen Teilaspekt des Unterrichts gelenkt werden. Der Unterricht soll nicht nur betrachtet, sondern in seiner Komplexität verstanden werden. Größere Zusammenhänge sollen nach der Reflexion erkannt werden. Der Beobachtungsbogen kann dieses Verstehen ermöglichen und so einen Beitrag zur verbesserten Lehrkompetenz leisten. Der geschulte Blick auf den Unterricht muss mit Blick auf die berufliche Zukunft immer wieder gefördert werden, auch wenn dies ein längerer Prozess ist.

Ebenso wie bei der Erstellung des Feedbackbogens gab es viele Vorlagen, die im Anhang zu finden sind. Je nachdem, welcher Aspekt des Unterrichts in den Fokus gerückt werden soll, werden bestimmte Kriterien hinzugefügt oder weggelassen. Die Erstellung des eigenen Instrumentes war auf die Bedürfnisse des Kurses zugeschnitten und konnte so den Blick auf für den Kurs Essentielles lenken. 
Auch bei diesem zweiten Projekt, der Entstehung des Beobachtungsbogens, wurden die eigenen Kompetenzen gefördert. Das fertige Produkt kann auch in Folgesemestern genutzt werden.

\section{FAZIT}

In diesem Artikel wurden zwei Instrumente vorgestellt, die durch den fortwährenden Austausch der Autorinnen im Rahmen eines Semesters entstanden sind. Bevor diese Reflexions- und Feedbackinstrumente detailliert vorgestellt wurden, wurden zunächst die beiden Seminare vorgestellt, die die Grundlage der entwickelten Instrumente bildeten. Für das Projektseminar ist der „Feedbackbogen - Kriterienkatalog für eine erfolgreiche Präsentation“ entstanden. Durch seine Allgemeingültigkeit konnte er allerdings auch in anderen Seminaren erfolgreich erprobt werden. Der „Beobachtungsbogen für den Unterricht" wurde im Rahmen eines Seminars, welches die Textkompetenz und Arbeit mit Texten thematisierte, erstellt. Bevor beide Instrumente ausführlich dargelegt und reflektiert wurden, wurden die Funktionen von Feedback und Beobachtungsbögen im Unterricht erläutert.

Insgesamt lässt sich festhalten, dass es eine lehrreiche und sinnvolle Erfahrung für die Praktikantin war, die ihre eigenen Kompetenzen und Reflexion durch die Erstellung und den Einsatz der Reflexionsinstrumente vertiefen konnte. Besonders der ständige Austausch und das Feedback ihrer Betreuerin Professor Dr. habil. Ilona Feld-Knapp trugen zum Lernzuwachs bei. Hervorzuheben ist weiterhin, dass sich die Instrumente durch die Diskussion und die Annäherung von Meinungen der Autorinnen entwickeln konnten. Für Frau Feld-Knapp haben die erstellten Reflexionsinstrumente, neben der Entwicklung ihrer Praktikantin, für die weitere Verwendung in zukünftigen Seminaren einen Nutzen. Aber auch die Studierenden konnten und können von den Ergebnissen profitieren, sie bekamen ein konstruktives Feedback und können den Bogen zum Überprüfen der eigenen Präsentationen nutzen oder um ein professionelles Feedback zu geben. Auch der Beobachtungsbogen kann in weiteren Seminaren für die Reflexion von Hospitationsstunden genutzt werden. 


\section{LITERATURVERZEICHNIS}

Bennewitz, H. (2012). Der Blick auf Lehrer/-innen. In: H. De Boer, S. Reh (Hrsg.), Beobachtung in der Schule - Beobachten lernen. Wiesbaden: Springer VS.

Brinitzer, M., Hantschel, H.-J., Kroemer, S., Möller-Frorath, M., Ros, L. (2016). DaF unterrichten. Basiswissen Didaktik. Deutsch als Fremd-und Zweitsprache. Stuttgart: Ernst Klett Sprachen.

Brunner, A., Armstrong, E. (2010). Feedback als Schlüsselelement einer neuen Lehr- und Lernkultur. Stuttgart/New York: Georg Thieme Verlag.

De Boer, H., Reh, S. (Hrsg.). (2012). Beobachtung in der Schule - Beobachten lernen. Wiesbaden: Springer VS.

Feld-Knapp, I. (2016). Textkompetenz und Aufgabenorientierung im Fremdsprachenunterricht. In: R. Freudenberg-Findeisen (Hrsg.), Aufdem Weg zu einer Textsortendidaktik. Linguistische Analysen und text(sorten)didaktische Bausteine nicht nur für den fremdsprachlichen Deutschunterricht. Hildesheim: Georg Olms Verlag.

Krumm, H.-J. (2010). Unterrichtsbeobachtung und Unterrichtsanalyse. In: H.-J. Krumm, Ch. Fandrych, B. Hufeisen, C. Riemer (Hrsg.), Deutsch als Fremd-und Zweitsprache. Ein internationales Handbuch. 2. Halbband. Berlin/New York: De Gruyter.

Noltenius, F. (2012). Evaluation, Feedback, Reflexion: Wie Schüler ihre Lehrer bewerten können. Hamburg: Diplomica Verlag.

Portmann-Tselikas, P., Schmölzer-Eibinger, S. (2008). Textkompetenz. Fremdsprache Deutsch, (39), 5-16.

Weber, H. (2012). Schülerinnen- und Schülerfeedback. „Instrumentenkoffer“. Abgerufen von www.lernvisionen.ch/kursunterlagen/downloads/sus-feedback-instrumentenkoffer.pdf

Summary: The article summarizes the cooperation of its authors and concerns instruments for reflection and their effective use in the context of university education. The tools presented are an assessment sheet containing the criteria for effective presentation and a class observation sheet, which is to assist those preparing for the teaching profession on their path to professional lesson observation. The article also discusses the various functions and importance of reflection in the learning process.

Keywords: feedback; lesson observation; project seminar; assessment criteria

Streszczenie: Artykuł stanowi podsumowanie współpracy autorek i dotyczy instrumentów służących refleksji oraz ich efektywnemu wykorzystaniu w kontekście kształcenia uniwersyteckiego. Prezentowanymi narzędziami są arkusz oceny zawierający kryteria skutecznej prezentacji oraz arkusz obserwacji zajęć, który ma wspomóc osoby przygotowujące się do zawodu nauczyciela na ich drodze do profesjonalnej obserwacji lekcji. W artykule omówiono także różnorodne funkcje i znaczenie refleksji w procesie kształcenia.

Słowa kluczowe: informacja zwrotna; obserwacja lekcji; seminarium projektowe; kryteria oceny 


\section{ANHANG}

\section{Erstellte Instrumente - Feedbackbogen}

\section{Feedbackbogen}

Kriterienkatalog für eine erfolgreiche Präsentation

\begin{tabular}{|c|c|c|c|c|c|c|}
\hline Inhalt & & ++ & + & / & - & -- \\
\hline Thema & $\begin{array}{l}\text { Passend gewählt, interessant, besonders, aktuell, } \\
\text { relevant, ... }\end{array}$ & & & & & \\
\hline Einleitung & $\begin{array}{l}\text { Ablauf klar zusammengefasst, Gründe für die } \\
\text { Themenwahl dargelegt, ... }\end{array}$ & & & & & \\
\hline Struktur/Aufbau & $\begin{array}{l}\text { Klar erkennbar, einfach zu folgen, logisch, roter } \\
\text { Faden, nachvollziehbar... }\end{array}$ & & & & & \\
\hline Qualität & $\begin{array}{l}\text { Fachlich richtig, Zusammenhänge erkennbar, } \\
\text { angemessene Gewichtung der Informationen, ... }\end{array}$ & & & & & \\
\hline Quantität & $\begin{array}{l}\text { Angemessen viele Informationen, Wesentliches } \\
\text { erarbeitet, auf Unwesentliches verzichtet, ... }\end{array}$ & & & & & \\
\hline Fragen & $\begin{array}{l}\text { Auf Fragen kann kompetent geantwortet werden, } \\
\text { sicher im Thema,... }\end{array}$ & & & & & \\
\hline
\end{tabular}

\begin{tabular}{|l|l|l|l|l|l|l|}
\hline Sprache und Auftreten & ++ & + & $/$ & - & -- \\
\hline Ausdrucksvermögen & $\begin{array}{l}\text { Variationsreich, differenziert, flexibel, fachlich } \\
\text { korrekt, korrekter Sprachgebrauch... }\end{array}$ & & & & & \\
\hline Sprechtempo & Klar, sicher, deutlich, ... & & & & & \\
\hline Redefluss & Freies Sprechen, flüssig, angemessene Pausen, ... & & & & & \\
\hline Gestik/Haltung & $\begin{array}{l}\text { Sicheres Auftreten, die Aussagen unterstützend, } \\
\text { locker ... }\end{array}$ & & & & \\
\hline Blickkontakt & Ansprechend, Publikum wird angesprochen, ... & & & & & \\
\hline
\end{tabular}

\begin{tabular}{|l|l|l|l|l|l|l|}
\hline Vermittlung & ++ & + & $/$ & - & -- \\
\hline Medieneinsatz & $\begin{array}{l}\text { Sinnvoll, ansprechende Gestaltung, routinierte } \\
\text { Anwendung, funktional... }\end{array}$ & & & & \\
\hline Kreativität & $\begin{array}{l}\text { Besondere Idee, Einsatz unterschiedlicher } \\
\text { Medien, ... }\end{array}$ & & & & \\
\hline Visualisierung & $\begin{array}{l}\text { Aussagekräftig, unterstützend, übersichtlich, } \\
\text { abwechslungsreich, ... }\end{array}$ & & & & \\
\hline Handout & $\begin{array}{l}\text { Übersichtlich, angemessen viele Informationen, } \\
\text { unterstützend zum Vortrag, ... }\end{array}$ & & & & \\
\hline Teamarbeit & Einheitliche Präsentation, klare Verteilung, ... & & & & \\
\hline
\end{tabular}

Notizen: 


\section{Erstellte Instrumente - Beobachtungsbogen}

\section{Beobachtungsbogen für den Unterricht}

\begin{tabular}{|c|c|c|c|c|c|}
\hline Kriterium & Beobachtungen und Ergänzungen & ++ & + & - & $\square$ \\
\hline $\begin{array}{l}\text { Unterrichtsklima und -führung } \\
\square \quad \text { Störungsfreie Arbeitsatmosphäre = viel Lern- } \\
\text { zeit } \\
\square \quad \text { Angemessenes Unterrichtstempo } \\
\square \quad \text { Konstruktiver Umgang mit Fehlern } \\
\square \quad \text { Freundlicher, rücksichtsvoller, respektvoller, } \\
\text { angstfreier Umgang miteinander } \\
\circ \quad \text { Lehrkraft (LK) \& SchülerInnen (SuS) } \\
\circ \quad \text { SuS untereinander }\end{array}$ & & & & & \\
\hline $\begin{array}{l}\text { Gesprächsformen und -gewichtung } \\
\square \quad \text { genutzte Gesprächsformen (Diskussion, } \\
\text { Frage/Antwort, Teamarbeit etc.) } \\
\square \quad \text { Jeder wird einbezogen } \\
\square \quad \text { Fördern von Sprechanlässen } \\
\square \quad \text { LK nimmt sich zurück } \\
\quad \text { LK-SuS-Gewichtung } \\
\square \quad \text { Eingehen auf Rück-/Verständnisfragen }\end{array}$ & & & & & \\
\hline $\begin{array}{l}\text { Zielorientierung/Struktur } \\
\square \quad \text { Klare Struktur der Informationen } \\
\square \quad \text { Präzise, nachvollziehbare Aufgabenstellungen } \\
\square \quad \text { LK drückt sich verständlich aus } \\
\square \quad \text { Angestrebte Lernziele werden deutlich } \\
\square \quad \text { Sinnvolle Übergänge }\end{array}$ & & & & & \\
\hline $\begin{array}{l}\text { Aktivierung und M otivierung } \\
\square \quad \text { Fördern der selbstständigen Bearbeitung von } \\
\text { Aufgaben, der aktiven Teilnahme } \\
\square \quad \text { Angemessene Leistungserwartungen } \\
\square \quad \text { Herausfordernde, abwechslungsreiche Lernan- } \\
\text { lässe } \\
\square \quad \text { Interessenorientierte, kompetenzfördernde } \\
\text { Themenwahl, kreative Umsetzung }\end{array}$ & & & & & \\
\hline $\begin{array}{l}\text { Textarbeit } \\
\square \quad \text { Einbezug unterschiedlicher Texte und Textsor- } \\
\text { ten } \\
\square \quad \text { Auswahl passender Texte - sprachliche und in- } \\
\text { haltliche Angemessenheit } \\
\square \quad \text { Vom Text zum Text - eigene Textproduktion } \\
\square \quad \text { Einbezug des 3-Phasen-Modells }\end{array}$ & & & & & \\
\hline $\begin{array}{l}\text { Variabilität der Unterrichtsformen } \\
\square \quad \text { Unterschiedliche methodische Vorgehenswei- } \\
\text { sen (Plenum, Gruppen-, Partner-, Einzelarbeit) } \\
\square \quad \text { Angemessene Medienvarianz }\end{array}$ & & & & & \\
\hline $\begin{array}{l}\text { Lernerfolgssicherung } \\
\square \quad \text { Besprechen von Ergebnissen } \\
\square \quad \text { Festhalten von Ergebnissen } \\
\square \quad \text { Sinnvolle, ergänzende Hausaufgaben }\end{array}$ & & & & & \\
\hline
\end{tabular}




\section{Feedbackbögen}

Feedbackbogen/Gütekriterien für eine Präsentation der Universität Göttingen. Abgerufen von www.google.com/url?sa $=t \& r c t=j \& q=\& e s r c=s \&$ source $=$ web $\& c d=1 \& v e d=2 a h U-$ KEwjJvvSOn6ffAhVK_aQKHRz-DOUQFjAAegQIChAC\&url=https\%3A\%2F\%2Fwww.uni-goettingen.de\%2Fde\%2Freferate\%253A\%2Bbewertungskriterien\%2F421479. html\&usg=AOvVaw3L1GcPHiuWXrw6_IJ_sitT

Bewertungskriterien für Referate/Vorträge/Seminargestaltung der Universität Jena. Abgerufen von www.personal.uni-jena.de/ s7oesv/bewertungskriterien.pdf

Bewertungsbogen für Referate der Freien Universität Berlin. Abgerufen von www.ewipsy.fu-berlin.de/einrichtungen/arbeitsbereiche/entwicklungswissenschaft/Lehre/ Hausarbeiten_Referate/Bewertungsbogen_Referate_Endversion_AB-Scheithauer_ Januar2016.pdf

Bewertungskriterien für die Präsentation des Oken-Gymnasiums Offenburg. Abgerufen von www.google.com/url?sa=t\&rct=j\&q=\&esrc=s\&source=web\&cd=1\&ved=2ahUKEwi2ku7eoKffAhXGzqQKHc-ZAdsQFjAAegQIChAC\&url=http\%3A\%2F\%2Foken.de\%2Fwp-content\%2Fuploads\%2F2008\%2F08\%2Fang_praesentation01. doc\&usg=AOvVaw19QnNDP4TpesEvrpJWqudT

Bewertungsbogen 2 der LehrerInnenfortbildung Baden-Württemberg. Abgerufen von lehrerfortbildung-bw.de/kompetenzen/projektkompetenz/bewertung/download/22DL_ Bew_Praesent_Bewertungsbogen2.doc

\section{Beobachtungsbögen}

Beobachtungsbogen für den Unterrichtsbesuch des Staatsinstituts für Schulqualität und Bildungsforschung. Abgerufen von www.praktikumsamt.mzl.uni-muenchen.de/materialien/u_beobachtungsbogen.pdf

BeobachtungsbogenfürdenUnterrichtdesStaatlichenSchulamts derStadtNürnberg. Abgerufen von www.google.com/url?sa=t\&rct=j\&q=\&esrc=s\&source=web\&cd=3\&ved=2ahUKEwiRg57guqnfAhVStHEKHRn_BBUQFjACegQIBxAC\&url=https\%3A\%2F\%2Fschulamt. info\%2Fmaterial\%2FKS00001_A\%2520_Beobachtungsbogen_Unterricht. doc\&usg=AOvVaw17OZd_niaXnqTx6poaLI4n

Beobachtungsbogen zur Reflexion von Unterricht gemäß des ORS des Bildungsservers Rheinland-Pfalz. Abgerufen von http://studienseminar.rlp.de/fileadmin/user_upload/ studienseminar.rlp.de/gs-web/Allgemeine_Infos/Beobachtungsbogen_ORS.pdf

Beobachtungsbogen zur Reflexion von Unterricht anhand der Kriterien des ORS des Bildungsservers Rheinland Pfalz. Abgerufen von http://studienseminar.rlp.de/fileadmin/_migrated/content_uploads/Beobachtungsbogen_laut_ORS_01.pdf

Zehn Beobachtungsbogen zu Einzelmerkmalen der Carl von Ossietzky Universität Oldenburg (Hilbert Meyer). Abgerufen von https://uol.de/fileadmin/user_upload/paedagogik/personen/hilbert.meyer/5.Zehn_Beobachtungsbogen_zu_Einzelmerkmalen. pdf 